\title{
Density dependence of nuclear symmetry energy constrained by mean-field calculations
}

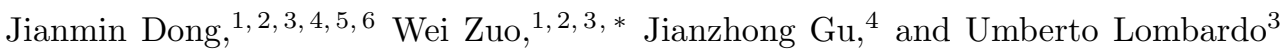 \\ ${ }^{1}$ Research Center for Nuclear Science and Technology, \\ Lanzhou University and Institute of Modern Physics of CAS, Lanzhou 730000, China \\ ${ }^{2}$ Institute of Modern Physics, Chinese Academy of Sciences, Lanzhou 730000, China \\ ${ }^{3}$ Dipartimento di Fisica and INFN-LNS, Via S. Sofia 64, I-95123 Catania, Italy \\ ${ }^{4}$ China Institute of Atomic Energy, P. O. Box 275(10), Beijing 102413, China \\ ${ }^{5}$ School of Nuclear Science and Technology, Lanzhou University, Lanzhou 730000, China \\ ${ }^{6}$ Graduate University of Chinese Academy of Sciences, Beijing 100049, China
}

(Dated: October 30, 2018)

\begin{abstract}
We establish a correlation for the symmetry energy at saturation density $S_{0}$, slope parameter $L$ and curvature parameter $K_{\text {sym }}$ based on widely different mean field interactions. With the help of this correlation and available empirical and theoretical information, the density dependent behavior around the saturation density is determined. We compare the results obtained with the present approach with those by other analyses. With this obtained density dependent behavior of the symmetry energy, the neutron skin thickness of ${ }^{208} \mathrm{~Pb}$ and some properties of neutron stars are investigated. In addition, it is found that the expression $S(\rho)=S_{0}\left(\rho / \rho_{0}\right)^{\gamma}$ or $S(\rho)=12.5\left(\rho / \rho_{0}\right)^{2 / 3}+$ $C_{p}\left(\rho / \rho_{0}\right)^{\gamma}$ does not reproduce the density dependence of the symmetry energy as predicted by the mean-field approach around nuclear saturation density.
\end{abstract}

PACS numbers: 21.65.Ef, 21.65.Cd, 26.60.Gj

\section{INTRODUCTION}

Our knowledge about the features of nuclear matter at saturation density $\rho_{0}$ is based primarily on the masses of nuclei, like the density $\rho_{0}=0.16 \mathrm{fm}^{-3}$, energy per particle $a_{v}=-16 \mathrm{MeV}$ and symmetry energy $S\left(\rho=\rho_{0}\right)=$ $28-34 \mathrm{MeV}$ [1]. However, the variation of the symmetry energy with density is still intensely debated [2 5]. The symmetry energy which characterizes the isospindependent part of the equation of state (EOS) of asymmetric nuclear matter, plays a crucial role in many issues of nuclear physics as well as astrophysics. It relates the heavy ion reactions [6 13], stability of superheavy nuclei [14], fusion cross sections [15] and structures, composition and cooling of neutron stars 16 19]. Many theoretical and experimental efforts have been performed to constrain the density-dependent symmetry energy [3]

The energy per particle of nuclear matter with density $\rho=\rho_{n}+\rho_{p}$ and asymmetry $\delta=\left(\rho_{n}-\rho_{p}\right) / \rho$ is usually written as $e(\rho, \delta)=e(\rho, 0)+S(\rho) \delta^{2}$, where $\rho_{n}, \rho_{p}$ and $\rho$ are the neutron, proton and nucleon densities. Around the nuclear matter saturation density $\rho_{0}$, the symmetry energy $S(\rho)$ can be expanded to second order in density as

$$
\begin{aligned}
S(\rho)= & S_{0}+\frac{L}{3}\left(\frac{\rho-\rho_{0}}{\rho_{0}}\right)+\frac{K_{\mathrm{sym}}}{18}\left(\frac{\rho-\rho_{0}}{\rho_{0}}\right)^{2} \\
& +\mathcal{O}\left[\left(\frac{\rho-\rho_{0}}{\rho_{0}}\right)^{3}\right],
\end{aligned}
$$

where $L=3 \rho \partial S(\rho) /\left.\partial \rho\right|_{\rho_{0}}$ and $K_{\text {sym }}=9 \rho^{2} \partial S^{2} /\left.\partial \rho^{2}\right|_{\rho_{0}}$ are the slope and curvature parameters at $\rho_{0}$ that govern the density dependence of $S(\rho)$ around $\rho_{0}$. $S_{0}$ describes the symmetry energy at density $\rho_{0}$. Recently, some progress has been made in determining the density dependence of $S(\rho)$ around the saturation density $\rho_{0}$ from nuclear isospin diffusion, double $\mathrm{n} / \mathrm{p}$ ratio in intermediate energy heavy-ion collisions, pygmy dipole resonance, neutron skin thickness and the nuclear binding energy. A description of isospin diffusion data with a symmetry energy of $S(\rho)=S_{0}\left(\rho / \rho_{0}\right)^{\gamma}$ with $\gamma=0.69-1.05$ has been obtained by using an isospin-dependent BoltzmannUehling-Uhlenbeck (IBUU) transport model 20], and a value of $\gamma=0.5$ is inferred from the preequilibrium neutron and proton transverse emissions comparing to IBUU transport calculations [21]. The giant dipole resonance (GDR) of ${ }^{208} \mathrm{~Pb}$ analyzed with the Skyrme interactions implies $\gamma=0.5-0.65$ [22]. The $S(\rho)$ extracted from more than 2000 measured nuclear masses gives $\gamma=0.6-0.8$ [23]. More recently, the analysis of isospin diffusion and double ratio data involving neutron and proton spectra by an improved quantum molecular dynamics transport model suggests $\gamma=0.4-1.05$ with $S(\rho)=12.5\left(\rho / \rho_{0}\right)^{2 / 3}+C_{p}\left(\rho / \rho_{0}\right)^{\gamma}[24]$. Although significant progress was made in determining the symmetry energy, it remains an open question nowadays.

It has been established that the neutron skin thickness $\Delta R_{n p}$, given by the difference of neutron and proton rootmean-square radii of heavy nuclei, correlates linearly with the slope $L$ around the saturation 25 30. Although the theoretical predictions on $S(\rho)$ with the current nuclear mean field methods and neutron skin thickness are extremely diverse, this correlation is universal in the realm of mean field theory as it is based on widely different nu- 
clear functionals [29, 30]. Based on the similar idea, in this work, we try to extract a relation of the three quantities $S_{0}, L$ and $K_{\text {sym }}$ in widely different mean field interactions to constrain the density dependent symmetry energy $S(\rho)$. This work is organized as follows. In Sec. II, we extract a relation between $S_{0}, L$ and $K_{\text {sym }}$ universally within the mean field framework since it is based on widely different nuclear mean-field interactions. In sec. III, by employing this relation and other considerations, we determine the density dependence of the symmetry energy around the saturation density. With the obtained density dependent behavior of the symmetry energy, the neutron skin thickness of ${ }^{208} \mathrm{~Pb}$ and some properties of neutron stars are investigated. Finally, a short summary is given in Sec. IV.

\section{ESTABLISHMENT OF THE RELATION FOR THREE QUANTITIES $S_{0}, L$ AND $K_{\text {sym }}$}

Let us first establish a relation of the three quantities. With the relation $S(\rho)=S_{0}\left(\rho / \rho_{0}\right)^{\gamma}$ describing the density dependence of the symmetry energy [20], one can obtain $L=3 S_{0} \gamma$ and $K_{\text {sym }}=9 S_{0} \gamma(\gamma-1)$, and thus a correlation of $S_{0}, L$ and $K_{\text {sym }}$ can be derived

$$
S_{0}=\frac{L}{3+K_{\mathrm{sym}} / L} .
$$

For the other density dependent behavior $S(\rho)=$ $12.5\left(\rho / \rho_{0}\right)^{2 / 3}+C_{p}\left(\rho / \rho_{0}\right)^{\gamma}$, we have $L=25+3\left(S_{0}-\right.$ $12.5) \gamma$ and $K_{\text {sym }}=-25+9 C_{p} \gamma(\gamma-1)$. Then, the correlation of $S_{0}, L$ and $K_{\text {sym }}$ takes the form

$$
S_{0}=12.5+\frac{(L-25)^{2}}{3 L+K_{\mathrm{sym}}-50} .
$$

The shape of the density dependence of the symmetry energy $S(\rho)$ that from the density-dependent M3Y (DDM3Y) interaction [32] can be written as $S(\rho)=$ $C_{k}\left(\rho / \rho_{0}\right)^{2 / 3}+C_{1}\left(\rho / \rho_{0}\right)+C_{2}\left(\rho / \rho_{0}\right)^{5 / 3}$ with $C_{k}=\left(2^{2 / 3}-\right.$ 1) $\frac{5}{3} \frac{\hbar^{2} k_{F 0}^{2}}{2 m}=13.0 \mathrm{MeV} . S_{0}, L$ and $K_{\text {sym }}$ can be expressed by parameters $C_{1}$ and $C_{2}$ with $S_{0}=13.0+C_{1}+C_{2}, L=$ $26.0+3 C_{1}+5 C_{2}$ and $K_{\mathrm{sym}}=-26+10 C_{2}$. Therefore, the correlation is given by

$$
S_{0}=2.6+\frac{L}{3}-\frac{K_{\mathrm{sym}}}{15} .
$$

We now test whether Eqs. (2), (3) and (4) work well or not by using widely different mean field functionals including relativistic and non-relativistic versions. As done in Ref. [31], to prevent eventual biases in our study, we avoid including more than two models of the same kind fitted by the same group. We also avoid models yielding a charge radius of ${ }^{208} \mathrm{~Pb}$ away from experiment data by more than $1 \%$ as in Ref. [31] since we will study the neutron skin thickness of ${ }^{208} \mathrm{~Pb}$. The interactions we used here are (1)LNS1, (2)LNS5, (3)MSL0,

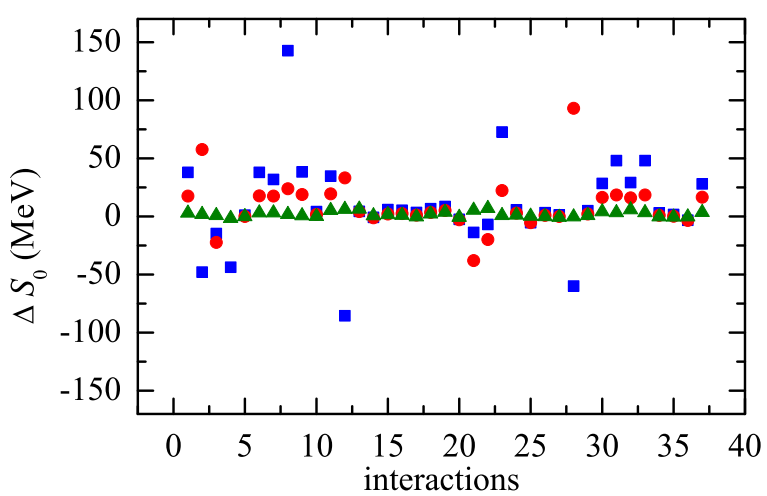

FIG. 1: (Color online) $\Delta S_{0}=S_{0}-L /\left(3+K_{\text {sym }} / L\right)$ (rectangle symbols $), \Delta S_{0}=S_{0}-12.5-(L-25)^{2} /\left(3 L+K_{\mathrm{sym}}-50\right)$ (circle symbols) and $\Delta S_{0}=S_{0}-2.6-L / 3-K_{\text {sym }} / 15$ (triangle symbols) within the density dependent behavior $S(\rho)=$ $S_{0}\left(\rho / \rho_{0}\right)^{\gamma}, S(\rho)=12.5\left(\rho / \rho_{0}\right)^{2 / 3}+C_{p}\left(\rho / \rho_{0}\right)^{\gamma}$ and DDM3Y shape, respectively. The horizontal ordinate denotes the sequence number for the interactions mentioned in text.

(4)SIV, (5)SkT4, (6)T6, (7)SkP, (8)SkM*, (9)SkX, (10)PK1, (11)D1S, (12)SLy4, (13)FSUGold, (14)SkMP, (15)SkI5, (16)NLSH, (17)TM1, (18)NL3, (19)NL1, (20)Sk255, (21)DDME1, (22)DDME2, (23)DDM3Y, (24)PC-F1, (25)Ska, (26)SV, (27)QMC, (28)MSkA, (29)SkI2, (30)MSk7, (31)HFB-17, (32)BSk8, (33)BSk17, (34)GM1, (35)GM3, (36)Sk272, (37)v090. The rootmean-square deviations given by Eqs. (2), (3) and (4) are $38.65 \mathrm{MeV}, 65.29 \mathrm{MeV}$ and $2.94 \mathrm{MeV}$ for $S_{0}$ value, respectively. In order to give a clearer show, we define a quantity $\Delta S_{0}$ to describe the differences between the left hand side and the right hand side of Eqs. (2), (3) and (4), and plot them in Fig. 1. As can be seen, $\Delta S_{0}$ with Eq. (44) approximates zero for these widely different interactions while $\Delta S_{0}$ with Eqs. (2), (3) tends to deviate from zero considerably. Therefore, Eqs. (2) and (3) can not be taken as accurate expressions for the description of the correlation of $S_{0}, L$ and $K_{\text {sym }}$ displayed by the mean-field calculations. It is noted that the minimum value of $K_{\text {sym }}$ is $-9 S_{0} / 4 \approx-72 \mathrm{MeV}$ with $S(\rho)=S_{0}\left(\rho / \rho_{0}\right)^{\gamma}$ and $-25-9 C_{p} / 4 \approx-69 \mathrm{MeV}$ with $S(\rho)=12.5\left(\rho / \rho_{0}\right)^{2 / 3}+C_{p}\left(\rho / \rho_{0}\right)^{\gamma}$. Many interactions, however, provide $K_{\text {sym }}<-100 \mathrm{MeV}$ (as will be seen in Fig. 2), which leads to large discrepancies of Eqs. (2) and (3) when compared to the mean-field predictions. This fact also indicates $S(\rho)=S_{0}\left(\rho / \rho_{0}\right)^{\gamma}$ or $S(\rho)=12.5\left(\rho / \rho_{0}\right)^{2 / 3}+C_{p}\left(\rho / \rho_{0}\right)^{\gamma}$ is not suitable to describe the density dependent behavior of the symmetry energy around $\rho_{0}$ as predicted by the mean field approach. In particular, they cannot describe a very soft symmetry energy due to their monotonous increase with 
the density. However, Eq. (44) is much better to be taken as a relationship for the correlation of $S_{0}, L$ and $K_{\text {sym }}$. Noting that the $S(\rho)$ with the DDM3Y shape is still approximate, Eq. (4) can be further improved to obtain the least deviation. Replacing the index $5 / 3$ in the DDM3Y shape by a coefficient $\gamma$, one has $S_{0}=a+L / 3+b K_{\text {sym }}$. By performing a least-squares fit with the calculated $S_{0}$, $L$ and $K_{\text {sym }}$ using the interactions above, the values of the parameters are $a=3.9199$ and $b=-0.07323$ with a rms deviation of $2.12 \mathrm{MeV}$ for $S_{0}$ value which is slightly different from that within the DDM3Y shape, and hence the relation is give by

$$
L=-11.76+3 S_{0}+\frac{K_{\mathrm{sym}}}{4.55},
$$

with a rms deviation of $6.35 \mathrm{MeV}$ for $L$ value. This formula can be considered as a universal one within the mean-field framework since it is based on widely different nuclear mean-field interactions. As a consequence, the analytical and simple expression reported in Eq. (5) describes in very good approximation the high correlations displayed between $S_{0}, L$ and $K_{\text {sym }}$ arising from the predictions of the representative set of employed meanfield models. The corresponding expression for the density dependence of the symmetry energy is given by

$$
S(\rho)=C_{k}\left(\frac{\rho}{\rho_{0}}\right)^{2 / 3}+C_{1}\left(\frac{\rho}{\rho_{0}}\right)+C_{2}\left(\frac{\rho}{\rho_{0}}\right)^{1.52},
$$

where $C_{k}=17.47$ is larger than that in the DDM3Y shape, which perhaps can be regarded as the mass in the kinetic energy being replaced by the effective mass $m^{*}$. In this step, parameters $C_{1}$ and $C_{2}$ remain unknown.

\section{DENSITY DEPENDENCE OF THE SYMMETRY ENERGY AROUND $\rho_{0}$ WITHIN THE OBTAINED RELATIONS}

TABLE I: The $S_{0}$ values obtained from various independent studies in other references.

\begin{tabular}{llll}
\hline \hline Reference & $S_{0}(\mathrm{MeV})$ & Reference & $S_{0}(\mathrm{MeV})$ \\
\hline Ref. [3, 20] & 31.6 & Ref. [23] & $31.1 \pm 1.7$ \\
Ref. [24] & 30.1 & Ref. [33] & $32.4 \pm 1.1$ \\
Ref. [34] & $32.0 \pm 1.8$ & Ref. [35] & 32.0 \\
Ref. [36] & $30.048 \pm 0.004$ & Ref. [4] & $32.3 \pm 1.3$ \\
\hline \hline
\end{tabular}

Of the three quantities $S_{0}, L$ and $K_{\mathrm{sym}}, S_{0}$ value is relatively well-known. Table I lists the recent $S_{0}$ value coming from recent various studies. The largest range of $S_{0}=31.6 \pm 2.2 \mathrm{MeV}$ from Table I will be used in the present study. Yet, we have to call for an additional condition to constrain the detailed $L$ and $K_{\text {sym }}$ values. Recently, Centelles et al. found that the symmetry energy (coefficient) $a_{\text {sym }}(A)$ of a finite nucleus with mass number $A$ is approximately equal to the symmetry energy $S\left(\rho_{A}\right)$ of nuclear matter at a reference density $\rho_{A}$,

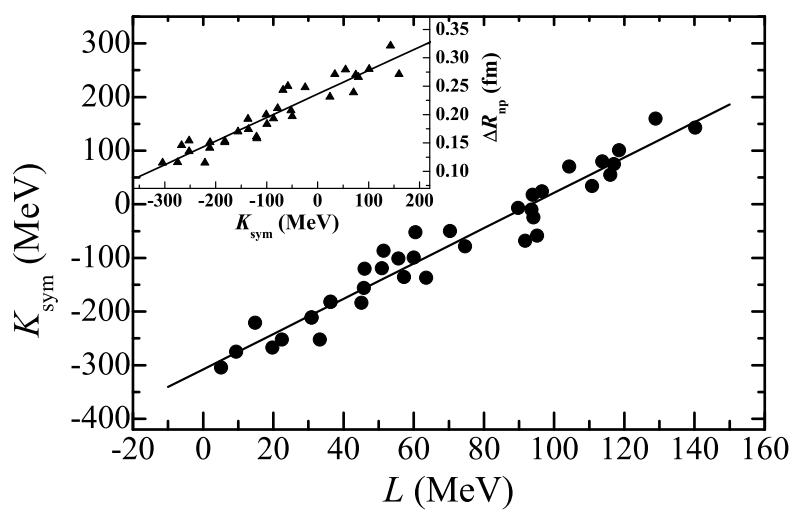

FIG. 2: Correlation of the curvature $K_{\text {sym }}$ with the slope $L$ of the symmetry energy at $\rho_{0}$. The line gives the fitting result with the correlation coefficient $r=0.972$. The inset displays the correlation between the neutron skin thickness $\Delta r_{n p}$ in ${ }^{208} \mathrm{~Pb}$ and $K_{\text {sym }}$ value. The line gives the fitting results with $r=0.945$.

namely, $S\left(\rho_{A}\right)=a_{\mathrm{sym}}(A)[29]$. For a given nucleus, the $\rho_{A}$ is determined, such as $\rho_{A}=0.1 \mathrm{fm}^{-3}$ for ${ }^{208} \mathrm{~Pb}$. Here, this relationship is employed to investigate the correlation between $K_{\text {sym }}$ and $L . a_{\text {sym }}(A)$ of a finite nucleus is given by

$$
a_{\mathrm{sym}}(A)=\frac{S_{0}}{1+\kappa A^{-1 / 3}}, \text { with } \kappa=\frac{9}{4} \frac{S_{0}}{Q},
$$

where $Q$ is the surface stiffness that measures the resistance of the nucleus against separation of neutrons from protons to form a neutron skin. In Ref. [30], it is shown that $S_{0} / Q$ ratio displays a linear relationship with $L$. Then, parameter $\kappa$ can be written as $\kappa=m L+n$. By combining Eq. (7) and the expression of the symmetry energy obtained with the DDM3Y shape aforesaid, the relationship of $S\left(\rho_{A}\right)=a_{\text {sym }}(A)$ can be converted into $L^{2}+a_{1} L K_{\mathrm{sym}}+a_{2} K_{\mathrm{sym}}+a_{3} L+a_{4}=0$ with new coefficients $a_{1}, a_{2}, a_{3}$ and $a_{4}$ by replacing $S_{0}, C_{1}$ and $C_{2}$ by $L$ and $K_{\text {sym }}$. We fit the results from the nuclear mean field calculations with the interactions mentioned above. However, in the fitting process, it is found that the first two terms can be neglected in the region under consideration. No matter whether or not the first two terms are taken into account, one can obtain the same root-meansquare deviation $\sqrt{\left\langle\sigma^{2}\right\rangle}=29.8 \mathrm{MeV}$ for $K_{\text {sym }}$ value. Accordingly, this correlation can be further simplified as a linear relation, as visibly shown in Fig. 2. By performing a two parameter fitting to this relationship, we have

$$
K_{\mathrm{sym}}=-307.862+3.292 L,
$$

with $\sqrt{<\sigma^{2}>}=29.8 \mathrm{MeV}$ for $K_{\text {sym }}$ value and the correlation coefficient $r=0.972$. The results of fitting are 
presented in Fig. 2 by the line. One readily sees that the predicted $K_{\text {sym }}$ with the mean field approaches varies largely from $-300 \mathrm{MeV}$ to $150 \mathrm{MeV}$. By combining Eq. (5) and Eq. (8) together with $S_{0}=31.6 \pm 2.2 \mathrm{MeV}$, in the present work, some information on the density dependence of $S(\rho)$ is investigated.

Fig. 3 illustrates the present estimated $L$ values compared with those from other independent approaches. One can find that the present finding has a remarkable overlap with but on the whole slightly softer than the very recent results of Carbone et al. [4], Liu et al. [23] and Tsang et al. [24]. This, to a large extent, perhaps stems from the relation of Eq. (5) based on the formula of $S(\rho)$ that can characterize the very soft symmetry energy in a much more reasonable manner. In the other analysis with $S(\rho)=S_{0}\left(\rho / \rho_{0}\right)^{\gamma}$ or $S(\rho)=12.5\left(\rho / \rho_{0}\right)^{2 / 3}+C_{p}\left(\rho / \rho_{0}\right)^{\gamma}$, the slope parameter of the symmetry energy at saturation density should be overestimated. To give a clearer explanation, we present an example here to show the overestimation of the $L$ values with $S(\rho)=S_{0}\left(\rho / \rho_{0}\right)^{\gamma}$. In Ref. [33], Danielewicz and Lee extracted the mass dependent symmetry energy coefficients of finite nuclei $a_{\text {sym }}(A)=S_{0}\left(1+\kappa A^{-1 / 3}\right)^{-1}$ with $S_{0}=27.39 \mathrm{MeV}$ and $\kappa=1.28$ [37]. With the relation $a_{\text {sym }}(A)=S\left(\rho_{A}\right)$ proposed in Ref. [29], if the density dependent behavior $S(\rho)=S_{0}\left(\rho / \rho_{0}\right)^{\gamma}$ is adopted, one obtains $L=34 \mathrm{MeV}$. However, if the DDM3Y shape or the modified one (Eq. (6) ) is applied, we obtain $L=21$ $\mathrm{MeV}$ and $L=24 \mathrm{MeV}$, respectively, lower than that stemming from $S(\rho)=S_{0}\left(\rho / \rho_{0}\right)^{\gamma}$. With $S_{0}=29.4-33.8$ $\mathrm{MeV}$ in Table I, the $L$ and $K_{\text {sym }}$ values obtained with the present method are $56 \pm 24 \mathrm{MeV}$ and $-125 \pm 79 \mathrm{MeV}$, respectively. It is interesting to see that the window reported in reference of Warda et al. [30] for the analysis of anti-protonic atoms data and that in the present manuscript are exactly the same, as shown in Fig. 3. The $S_{0}$ value with less uncertainty will lead to narrower windows of $L$ and $K_{\text {sym }}$ values within our approach. The errors for $L$ and $K_{\text {sym }}$ arising from the errors of the parameters in the fitting are less important compared with these resulting from the uncertainty of $S_{0}$ value. Therefore, for simplicity and clarity, we only selected the optimal fitting results. The small deviation and large correlation coefficient indicate that the parameters are well constrained by the information used in the fitting procedure.

It is well known that $L$ shows a linear relationship with neutron skin thickness $\Delta R_{n p}$ of finite nuclei. In Ref. [31], this linear correlation is given by $\Delta R_{n p}=0.101+$ $0.00147 L$ for ${ }^{208} \mathrm{~Pb}$. Because of Eq. (8),$K_{\text {sym }}$ should also show a linear correlation with the neutron skin thickness $\Delta R_{n p}$. Using the aforesaid interactions for finite nuclei, we obtain the correlation between $\Delta R_{n p}$ in ${ }^{208} \mathrm{~Pb}$ and $K_{\text {sym }}$ by performing a fitting procedure

$$
\begin{aligned}
\Delta R_{n p}= & (0.236210 \pm 0.003991) \\
& +(0.000415 \pm 0.000026) K_{\mathrm{sym}},
\end{aligned}
$$

where $\Delta R_{n p}$ and $K_{\mathrm{sym}}$ are in units of fm and $\mathrm{MeV}$, re-

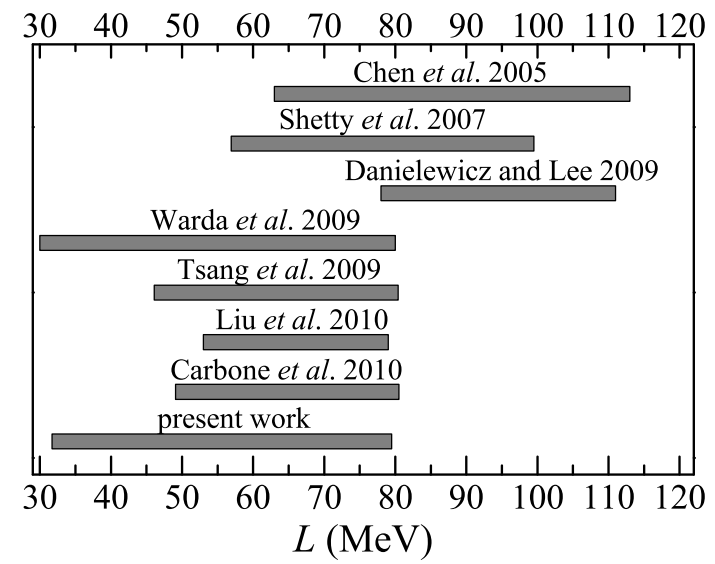

FIG. 3: Comparison between the $L$ values obtained in the present work and those from other recently various analyses including Carbone et al. [4], Liu et al. [23], Tsang et al. 24], Warda et al. 30], Danielewicz and Lee 33], Shetty et al. 3] and Chen et al. [20].

spectively. The result of the fitting is presented in the inset of Fig. 2 by the line. A larger $K_{\text {sym }}$ value implies a thicker neutron skin. The linear relation allows one to extract $K_{\text {sym }}$ with the measured $\Delta R_{n p}$. Therefore, once an accurate measurement of $\Delta R_{n p}$ is achieved, $L$ as well as $K_{\text {sym }}$ values can be determined simultaneously. As a consequence, a richer information about the density dependent behavior of the symmetry energy can be achieved. Here $K_{\text {sym }}$ is used in turn to determine the $\Delta R_{n p}$. With the $K_{\text {sym }}$ values obtained above with $S_{0}=31.6 \pm 2.2 \mathrm{MeV}$, the $\Delta R_{n p}$ in ${ }^{208} \mathrm{~Pb}$ is estimated to be $0.185 \pm 0.035 \mathrm{fm}$. With the relationship between $\Delta R_{n p}$ and $L$ in Ref. 31] together with the $L$ values of $56 \pm 24 \mathrm{MeV}$ that we obtained above, the $\Delta R_{n p}$ for ${ }^{208} \mathrm{~Pb}$ is $0.183 \pm 0.035 \mathrm{fm}$, which is quite consistent with that from $\Delta R_{n p}-K_{\mathrm{sym}}$ correlation. This fact indicates the justification of our approach to a large extent. Again, our calculated $\Delta R_{n p}$ for ${ }^{208} \mathrm{~Pb}$ is in good agreement with the value from analysis of PDR [4, 34]. The neutron-rich skin of a heavy nucleus is related to the properties of neutron star crusts. For instance, the thicker the neutron skin is, the thinner the solid crust of a neutron star [16]. Horowitz and Piekarewicz proposed that if $\Delta R_{n p}$ for ${ }^{208} \mathrm{~Pb}$ is greater than about $0.24 \mathrm{fm}$, the electron fraction becomes large enough to allow the direct URCA process to cool down a $1.4 M_{\odot}$ neutron star [16]. Our calculated $\Delta R_{n p}$ is too small to allow the direct URCA process in this canonical neutron star to occur. An almost linear relationship between the $\Delta R_{n p}$ and the critical density $\rho_{c}$ of a phase transition from nonuniform to uniform neutron rich matter is put forward in Ref. [16] with an approximate relation of $\rho_{c} \approx 0.16-0.39 \Delta R_{n p}\left({ }^{208} \mathrm{~Pb}\right)$. 
Using this relation combining with $\Delta R_{n p}$ in ${ }^{208} \mathrm{~Pb}$ deduced above, one obtains $\rho_{c} \approx 0.09 \mathrm{fm}^{-3}$ which is in accord with $\rho_{c} \approx 0.096 \mathrm{fm}^{-3}$ of the microscopic EOS of Friedman and Pandharipande [38]. These two examples display the applications of the correlations we employed in understanding the physics of compact objects.

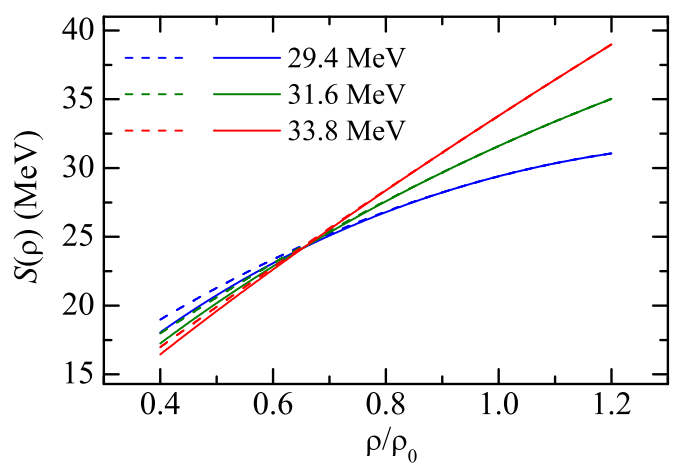

FIG. 4: (Color online) Behavior of the density dependent symmetry energy with different $S_{0}$ values from Eqs. (1) (dash lines) and (6) (solid lines), respectively.

When $S_{0}$ value is fixed, the parameters $C_{1}$ and $C_{2}$ in Eq. (6) are accordingly determined, and hence the density dependence of the symmetry energy in the form of a modified DDM3Y shape is obtained. Within this density dependent behavior of $S(\rho)$, one may investigate the symmetry energy at subsaturation densities. Fig. 4 presents the symmetry energy $S(\rho)$ as a function of the density with several detailed $S_{0}$ values. The solid (dash) curves denote the calculations with Eq. (6) (Eq. (1)). As can be seen, $S(\rho)$ shows different behaviors with different $S_{0}$ values. The symmetry energy tends to become stiffer with a larger $S_{0}$ value. When $S_{0}$ is selected to be 33.8 $\mathrm{MeV}$, the relation of $S(\rho)-\rho$ is almost linear. This explains the fact that the relativistic mean field models have traditionally predicted a stiff symmetry energy-these interactions give larger $S_{0}$ values. The density dependent behavior stemming from Eq. (6) and Taylor expansion of Eq. (1) mostly coincide with each other, which indicates that the Taylor expansion of Eq. (1) can be applied in a wide density range when Eq. (6) is employed for the description of $S(\rho)$. In addition, consistent with the meanfield results of Refs. [25, 27, 29], it is found that different curves give nearly the same value for the symmetry energy at a density around $\rho=0.1 \mathrm{fm}^{-3}$. The obtained symmetry energy $S(\rho)=23.6 \pm 0.02 \mathrm{MeV}$ at a nucleon density of $\rho=0.1 \mathrm{fm}^{-3}$ agrees with $23.3-24.9 \mathrm{MeV}$ by an analysis of the GDR of ${ }^{208} \mathrm{~Pb}$ with the Skyrme interactions [22] but is larger than the values of $21.2-22.5$ $\mathrm{MeV}$ obtained with analysis of the GDR of ${ }^{132} \mathrm{Sn}$ within relativistic mean field models 39]. Moreover, compared with that from the PDR, our result includes a much less uncertainty. As suggested by Daoutidis and Goriely [40], the PDR strength measurements nowadays cannot yield quantitative insight on the symmetry energy, and thus both theoretical approaches as well as phenomenological interactions need to be further improved to investigate the symmetry energy. Some authors even proposed that the PDR strength is weakly correlated with the neutron skin thickness of heavy nuclei [41] and hence the density dependence of the symmetry energy. Finally, we would note that compared with the transport models and the PDR measurements, our approach is much more straightforward. Yet, our approach is based only on mean field models and could only gain some limited information about the symmetry energy. Opposite to our method, the analysis of the experimental data within microscopic techniques, such as the random phase approximation for the study of the PDR, may allow one to investigate the internal structure and dynamics of the nucleus, and hence one can obtain more detailed knowledge. These different approaches can validate or complement each other to get more compelling results.

\section{SUMMARY}

Based on the similar idea in Ref. [25 30] that the neutron skin thickness correlates with the slope parameter $L$ around the saturation density in mean field models, we have established a relation for three quantities $S_{0}$, $L$ and $K_{\text {sym }}$ in widely different mean field interactions. With this relation and other constraint conditions, the density dependence of the nuclear symmetry energy $S(\rho)$ has been investigated in the present work. With the obtained density dependence of the symmetry energy, the neutron skin thickness of ${ }^{208} \mathrm{~Pb}$ and some properties of neutron stars were analyzed. The main conclusions are as follows. (i) It is not suitable to take the form of $S(\rho)=S_{0}\left(\rho / \rho_{0}\right)^{\gamma}$ or $S(\rho)=12.5\left(\rho / \rho_{0}\right)^{2 / 3}+C_{p}\left(\rho / \rho_{0}\right)^{\gamma}$ to describe the behavior of the symmetry energy as predicted by the mean-field approach around the nuclear saturation density while the shape from the DDM3Y is much better. (ii)Based on the latter formula, we have provided an analytical and simple expression for the high correlation shown by $S_{0}, L$ and $K_{\text {sym }}$ within the mean field framework. Therefore, if the symmetry energy at saturation density $\rho_{0}$ is known, the values of $K_{\text {sym }}$ and $L$ can be unambiguously related by using Eq. (5). The $L$ and $K_{\text {sym }}$ values in the present study are $56 \pm 24 \mathrm{MeV}$ and $-125 \pm 79 \mathrm{MeV}$ respectively with $S_{0}=31.6 \pm 2.2 \mathrm{MeV}$ yielded in other references.(iii) The neutron skin thickness of ${ }^{208} \mathrm{~Pb}$ displays a linear correlation with $K_{\text {sym }}$. Thus, once the neutron skin thickness is measured accurately, not only slope parameter $L$ but also curvature parameter $K_{\text {sym }}$ of the symmetry energy around the saturation can be determined. Thus, a richer information about the density dependent behavior of the symmetry energy can be achieved. $\Delta R_{n p}$ of ${ }^{208} \mathrm{~Pb}$ is $0.185 \pm 0.035$ fm with the $K_{\text {sym }}$ value in item (ii) which is too small 
to allow the direct URCA process in $1.4 M_{\odot}$ neutron star to occur.(iv) Within our approach, the behavior of $S(\rho)$ against $\rho$ around the normal density almost relies on the symmetry energy at saturation density $\rho_{0}$. We show that a large $S_{0}$ value leads to a stiff symmetry energy. They, however, naturally provide almost the same result of about $23.6 \mathrm{MeV}$ at $\rho=0.1 \mathrm{fm}^{-3}$.

\section{ACKNOWLEDGMENTS}

This work was supported by the National Natural Science Foundation of China (with Grant Nos.
$11175219,10875151,10975190,11075066,11175074), \quad$ the Knowledge Innovation Project (KJCX2-EW-N01) of Chinese Academy of Sciences, the Chinese Academy of Sciences Visiting Professorship for Senior International Scientists (Grant No.2009J2-26), CAS/SAFEA International Partnership Program for Creative Research Teams (CXTD-J2005-1), and the Funds for Creative Research Groups of China under Grant No. 11021504.
[1] P. Danielewicz, Nucl. Phys. A727, 233 (2003).

[2] D. Vretenar and T. Niksić and P. Ring, Phys. Rev. C 68,024310 (2003).

[3] D. V. Shetty, S. J. Yennello, and G. A. Souliotis, Phys. Rev. C 75, 034602 (2007), and references therein.

[4] Andrea Carbone et al., Phys. Rev. C 81, 041301(R) (2010).

[5] J. Zenihiro et al., Phys. Rev. C 82, 044611 (2010).

[6] P. Danielewicz, R. Lacey, and W. G. Lynch, Science 298, 1592 (2002).

[7] V. Baran, M. Colonna, V. Greco, and M. Di Toro, Phys. Rep. 410, 335 (2005).

[8] B. A. Li, L. W. Chen, and C. M. Ko, Phys. Rep. 464, 113 (2008).

[9] J. M. Lattimer and M. Prakash, Phys. Rep. 442, 109 (2007).

[10] Sanjeev Kumar, Y. G. Ma, G. Q. Zhang and C. L. Zhou, Phys. Rev. C 84, 044620 (2011).

[11] Zhao-Qing Feng, Phys. Rev. C 83, 067604 (2011).

[12] Gao-Chan Yong, Phys. Rev. C 84, 014607 (2011).

[13] Chun-Wang Ma, Fang Wang, Yu-Gang Ma, Chan Jin, Phys. Rev. C 83, 064620 (2011).

[14] Jianmin Dong, Wei Zuo, and Werner Scheid, Phys. Rev. Lett. 107, 012501 (2011).

[15] C. Rizzo, V. Baran, M. Colonna, A. Corsi, and M. Di Toro, Phys. Rev. C 83, 014604 (2011).

[16] C. J. Horowitz and J. Piekarewicz, Phys. Rev. Lett. 86, 5647 (2001).

[17] J. M. Lattimer and M. Prakash, Phys. Rep. 333, 121 (2000); Science 304, 536 (2004).

[18] B. G. Todd-Rutel and J. Piekarewicz, Phys. Rev. Lett. 95, 122501 (2005).

[19] R. Cavagnoli, D. P. Menezes and C. Providencia, Phys. Rev. C 84, 065810 (2011).

[20] Lie-Wen Chen, Che Ming Ko, and Bao-An Li, Phys. Rev. Lett. 94, 032701 (2005); Phys. Rev. C 72, 064309 (2005); Bao-An Li and Lie-Wen Chen, Phys. Rev. C 72, 064611 (2005).
[21] M. A. Famiano et al., Phys. Rev. Lett. 97, 052701 (2006).

[22] L. Trippa, G. Colò, and E. Vigezzi, Phys. Rev. C 77, 061304(R) (2008).

[23] M. Liu, N. Wang, Z. X. Li, and F. S. Zhang, Phys. Rev. C 82, 064306 (2010).

[24] M. B. Tsang et al., Phys. Rev. Lett. 102, 122701 (2009).

[25] B. A. Brown, Phys. Rev. Lett. 85, 5296 (2000).

[26] S. Typel and B. A. Brown, Phys. Rev. C 64, 027302 (2001).

[27] R. J. Furnstahl, Nucl. Phys. A706, 85 (2002).

[28] A. W. Steiner, M. Prakash, J. Lattimer, and P. J. Ellis, Phys. Rep. 411, 325 (2005).

[29] M. Centelles, X. Roca-Maza, X. Vinas, and M. Warda, Phys. Rev. Lett. 102, 122502 (2009).

[30] M. Warda, X. Vinas,X. Roca-Maza, and M. Centelles, Phys. Rev. C 80, 024316 (2009).

[31] X. Roca-Maza, M. Centelles, X. Vinas, and M. Warda, Phys. Rev. Lett. 106, 252501 (2011).

[32] T. Mukhopadhyay, D. N. Basu, Nucl. Phys. A789 201 (2007).

[33] P. Danielewicz and J. Lee, Nucl. Phys. A818, 36 (2009).

[34] A. Klimkiewicz et al., Phys. Rev. C 76, 051603(R) (2007).

[35] H. Heiselberg and M. Hjorth-Jensen, Phys. Rep. 328, 237 (2000).

[36] T. Mukhopadhyay and D. N. Basu, Acta Phys. Pol. B 38, 3225 (2007).

[37] Ning Wang and Min Liu, Phys. Rev. C 81, 067302 (2010).

[38] C. P. Lorenz, D. G. Ravenhall, and C. J. Pethick, Phys. Rev. Lett. 70, 379 (1993).

[39] L. G. Cao and Z. Y. Ma, Chin. Phys. Lett. 25, 1625 (2008).

[40] I. Daoutidis and S. Goriely, Phys. Rev. C 84, 027301 (2011).

[41] P.-G. Reinhard and W. Nazarewicz, Phys. Rev. C 81, 051303(R) (2010). 